\title{
Public Engagement - More than just fun
}

\author{
Greg Corbett ${ }^{1, *}$, Ian Collier ${ }^{1}$, and Sophy Palmer ${ }^{1}$ \\ ${ }^{1}$ Science and Technology Facilities Council, Scientific Computing, Didcot, United Kingdom
}

\begin{abstract}
Public Engagement (PE) with science should be more than "fun" for the staff involved. PE should be a strategic aim of any publically funded science organisation to ensure the public develops an understanding and appreciation of their work, its benefits to everyday life and to ensure the next generation is enthused to take up STEM careers. Most scientific organisations do have aims to do this, but very few have significant budgets to deliver this. In a landscape of ever tightening budgets, how can we develop a sustainable culture of PE within these organisations?
\end{abstract}

UKRI/STFC's Scientific Computing Department present how we have worked to embed a culture of PE with the department by developing our early career staff members; highlighting the impact PE makes at the departmental and project level; and linking PE to our competency framework.

\begin{abstract}
We will also discuss how our departmental work interacts with and complements STFC's organisationalwide PE effort, such as making use of a shared evaluation framework that allows us to evaluate our public engagement activities against their goals and make strategic decisions about the programme's future direction.
\end{abstract}

\section{Introduction}

The Science and Technology Facilities Council (STFC), part of UK Research and Innovation (UKRI), is responsible for supporting, co-ordinating and promoting research, innovation and skills development in seven distinct fields [1]. STFC runs multiple high-tech laboratories, world-leading astronomical observatories, pioneering Science and Innovation Campuses, and state-of-the-art research facilities extending boundaries in laser science, computing, neutron-scattering and other disciplines [2]. Public Engagement is one of STFC's six strategic themes for 2010 to 2020 [3] and STFC also has a dedicated public engagement team with managers at each of the three largest sites. "Big Data and Computing" is one of their five key outreach themes [4].

STFC's Scientific Computing Department (SCD) is distributed across two STFC sites and manages high performance computing facilities, services and infrastructure, supporting some of the UK's most advanced scientific facilities. SCD works across a number of large facilities at both the Daresbury Laboratory in Cheshire and the Rutherford Appleton Laboratory in Oxfordshire (such as the UK Worldwide LHC Computing Grid (WLCG) Tier 1 [5] and the JASMIN Super Data Cluster [6]), and has close links with other computing centres around the UK, including the Hartree Centre $[7,8]$.

In 2013, SCD started to fund a public engagement committee so that it could play a fuller role in the computing outreach of STFC and increase the amount undertaken, without impacting other outreach themes. This paper presents how SCD have worked to embed a culture of PE within the department and how those efforts interact with the efforts of STFC.

\section{Public Engagement as a Strategy Priority}

PE should be a strategic priority of any publically funded STEM organisation to ensure the public develops an understanding and appreciation of their work and its benefits to everyday life. PE also helps to raise the aspirations of the next generation and ensure they feel enthused and capable to take up the STEM careers which are vital for society as, for example, nearly $40 \%$ of UK employers report difficulties recruiting staff with relevant STEM skills [9].

At STFC, "Inspiring and Involving" is one of six strategic themes that focusses delivery of its goals [10]. At CERN, for which STFC funds the UK contribution, "Inspiration and Education" is one of the four themes that form their

\footnotetext{
* Corresponding author: greg.corbett@stfc.ac.uk
} 
communication strategy [11]. Indeed, most publically funded scientific organisations do have PE as a high level aim, but very few have significant budgets to deliver this. This paper presents the work of UKRI/STFC's Scientific Computing Department (SCD) to embed a culture of PE within the department.

\section{Why staff do Pubic Engagement?}

Staff do Public Engagement (PE) for a multitude of reasons. Some staff do it for social good. Many STFC staff are able to recall being inspired by the Computing and STEM public engagement programmes of other institutions as a child and teenager and as such understand the importance of these activities, with PE providing the opportunity to "give back". Some staff do PE for the corporate good of their organisation - feeling that science organisations spending public money are accountable to the public and have a responsibility to engage the public with the work, or using PE as an opportunity to develop their professional skill set, raise the public profile of their organisation, and as a means of widening the STEM pipeline and meet long-term recruitment goals. Ultimately, most staff do it because it is fun! In recent years, the wellbeing of STFC's staff has become a corporate priority, and taking part in a session of PE can contribute to the good mental health and wellbeing of staff.

\section{STFC's Public Engagement Strategy}

STFC has a strong record in public engagement, using its work, national laboratories and campuses in Harwell, Daresbury, and Edinburgh to enable the public to experience the remarkable scale, ambition, and achievement of global science today [12]. The PE Strategy is delivered by STFC PE teams local to the three largest sites (who rely heavily on STEM staff volunteering time to deliver activities, workshops and tours) and a PE team at STFC headquarters (who coordinate national grants and projects). In a landscape of ever tightening budgets, the PE strategy concentrates this effort where it is most effective, by setting out five key aims.

\subsection{Showcasing STFC science and technology}

Experience shows that the public wants to see STFC's science and technology for themselves. To that end, STFC has welcomed tens of thousands of people, including thousands of school students and teachers, through the doors of its facilities in the past five years, receiving overwhelmingly positive feedback [12]. The strategy focusses STFC's PE efforts to within STFC's remit and further prioritises selected campaigns of strategic importance [12].

\subsection{Building the right partnerships}

STFC is recognised and sought-after as a leader in STEM engagement who prioritise the quality, reach, and impact of their engagement over the quantity, even if this means they deliver fewer activities themselves [12]. To achieve this, STFC set up a Legacy Award Scheme [13]. Legacy Awards encourage successful PE practitioners who have received STFC funding in the past to continue to grow and evolve their engagement over time, whilst developing a community network of highly-skilled practitioners and working with partners to secure the future of the engagement [13].

\subsection{Developing and supporting STEM influencers}

STFC considers STEM 'influencers' to be individuals working in a professional or voluntary capacity that are skilled in engaging their specific target audiences and are inspirational communicators who readily pass their passion for STEM onto those around them. As well as teachers and professional science communicators, STFC recognises the role its own staff and the researchers it funds play as STEM influencers and seeks to raise the public engagement skills of its research community by providing appropriate training and networking opportunities [12].

\subsection{Improving our reach with diverse audiences}

STFC's PE strategy states that there must be no barriers to engaging with STFC based on gender, ethnicity, social background, geography, or any other factor [12]. However, STFC understands that there are areas all across the UK with low science capital [14], where people don't have the same opportunities to explore science and technology, and feel that it's not "for them". STFC works with experienced partners to ensure an increasing proportion of their activities reach formerly underserved communities, giving them an equal voice by listening, understanding, and responding to what people want to know about science and technology [15].

\subsection{Delivering high quality public engagement activities and outcomes}

For STFC, delivering the highest-quality and maximising the likelihood of positive impacts from public engagement is vital. To improve their programme on a continuous basis, STFC focuses heavily on evaluation and has adopted a 
number of generic learning outcomes (GLOs) for their engagement activities, supported by underlying evaluation metrics and data collection approaches [12].

\section{Public Engagement in STFC's Scientific Computing Department}

Within STFC, organisational adoption of PE varies from department to department. In some departments, there is relatively little structural support for engagement. Those departments rely solely on the efforts of the STFC PE teams to engage the public with the work they do, although there is individual effort from staff volunteers within those departments. Other departments have dedicated staff responsible for managing their PE programme, although the delivery of this programme again relies on effort from staff volunteers.

SCD has its own director-endorsed PE strategy which further concentrates the department's effort to four main target audiences where SCD feel they can have the biggest impact: teachers of decision making students - i.e. those teaching students deciding on GCSEs, A-Levels and careers/further education; decision making students themselves; primary school children and the public in general. Rather than a dedicated PE Manager, SCD has a PE committee, made up of STEM staff from across the department and the STFC PE teams, to provide ongoing strategic direction and guidance to the department's PE programme. This committee leads on: raising the profile of computing with young people; providing teachers with the skills and support needed to develop engaging lessons and activities for their classes and increasing staff participation in PE activities. The committee has a yearly budget of $£ 10,000$ to enable it to achieve these aims.

\subsection{Interactions with STFC Public Engagement Teams}

The Public Engagement activities undertaken by SCD are well documented [16-19]. These activities do not happen in isolation, they interact with and complement STFC's organisational-wide PE effort.

A typical example of this is that SCD will lead on a series of hour long computing workshops and data centre tours as part of a wider event on site, while the STFC PE teams coordinate and manage the logistics of having visitors at the laboratory. Sometimes, these events are general STEM events without a direct computing theme, but these events are still important to SCD as they serve as an opportunity to highlight how computing underpins all of the work that STFC undertakes. SCD also leads on longer events as well, such as day-long Arduino workshops or week-long coding challenges, again relying on the STFC PE teams to manage the logistics.

SCD funds the development and delivery of new computing related public engagement workshops and events, which then go on to become staples of the STFC-wide PE programme. For example, the development of SCD's Arduino and LEGO Mindstorms workshops were undertaken by SCD graduates on a three month placement with the RAL Public Engagement team, whilst the necessary hardware and consumables to run these events regularly, as part of the wider STFC PE programme, is funded by the SCD PE committee's budget.

\subsection{Using shared STFC frameworks}

STFC's Public Engagement Strategy is unambiguous about the importance of evaluating public engagement in order to report on, improve and celebrate activities. STFC has produced an evaluation framework to evaluate their individual activities as well as their programme as a whole. This framework is based on the concept of "Generic Learning Outcomes" (GLOs), defined as "a response by the audience that we intend our public engagement to elicit, demonstrating learning in its broadest sense". The GLO model is widely used in the science centre and museums sector [20].

The SCD PE committee has adopted this framework. For each computing activity, SCD has defined the intended outcomes that the activity should produce, as the first step to evaluating and continuously improving our programme. These outcomes are defined in terms of what activity participants will do, feel, value, understand and have the skills to do later.

To develop a toolkit for the ongoing evaluation and improvement of the SCD PE programme, the SCD PE committee has commissioned an external evaluation project. The project covers four areas:

- Content: Do the existing activities achieve their intended outcomes, as defined by the GLOs? How can they be improved in the future?

- Audience: Who are SCD engaging with? Is this the target audience or is SCD's audience those with high science capital, who are already engaged with computing? What is the depth of audience engagement?

- Staff: Who volunteers (and does not volunteer) to do PE within SCD, and why? How can the SCD PE Committee increase participation across the department? How can staff review and improve their PE skills and workshops on an ongoing basis?

- Programme: Is the SCD PE programme as a whole meeting the strategic objectives set out in the SCD PE strategy? What elements of the strategy are working and which elements need changing?

\subsection{Training new staff}

SCD has a core group of dedicated researchers who regularly volunteer to help with the SCD Public Engagement programme. STFC's existing evaluation data consistently highlights the value students and the public place on engaging 
with 'real' computer scientists, and the participation of SCD staff in public engagement is a key feature of the STFC PE programme. The biggest limiting factor on the amount of PE that SCD can deliver is the number of staff willing and able to volunteer. One of the ways SCD addresses this issue is by training early career new starters across STFC, such as graduates and apprentices, to deliver PE. SCD focuses on this subset of its staff because:

- Generally, they have the time and management buy-in to deliver significant amounts of engagement. For graduates at STFC, their PE track record often forms part of their case for promotion at the end of the graduate scheme.

- They are closer to SCD's target age group for PE, so it's easier for the schoolchildren to see themselves in those staff members.

- Getting staff on board early means that they are more likely to make the time in future when they get busier, and to encourage people they subsequently manage to get involved in PE.

Training graduate new starters to act as Data Centre tour guides has become systematic in recent years, with all graduates in SCD receiving the necessary training. The graduates then spend their first year shadowing more experienced tour guides, allowing for an exchange of best practices.

Previously, training SCD staff to help with computing workshops was mostly an informal process - usually on a oneto-one, as needed, basis. This was necessary at first to build capacity within the department for PE. Because of this, SCD now has several staff members capable of running computing workshops, and this allows for a more systematic approach to workshop training going forward. In November 2019, SCD trialled a Computing PE Induction Day with the aim of enabling new computing graduates and apprentices across STFC to get up to speed and involved more quickly and effectively than in previous years. $45 \%$ of the attendees to this induction were STFC computing staff outside SCD, reflecting the fact that other STFC departments support the computing engagement that SCD leads on.

The day started with an introduction to "why PE is important" from a member of SCD's senior leadership team and "high quality PE" from a member of the RAL PE Team. This endorsement of Public Engagement from senior staff is important, as it demonstrates SCD's commitment. This was then followed by a series of "train the trainer" style introductions to SCD's three core PE workshops; a BeeBot activity, an Arduino activity and a LEGO Mindstorms activity. The day ended with an overview of the many other ways one can get involved with SCD's PE efforts, such as being a work experience supervisor, supporting the coding club at RAL and developing new material. This induction was the first SCD has run of its kind, and as such evaluating its effectiveness is very important to determine if it should be repeated and if it should have a wider participant pool than early career new starters. Initial evaluation, gathered via an e-survey immediately after the workshop, showed that ten of the thirteen respondents (out of a total of twenty attendees) said they felt confident to help with all three of SCD's core PE workshops. The initial evaluation also showed the respondents felt the event was enjoyable and well run, giving the workshop an average overall score of $4.8 / 5$. Further evaluation of the workshop will be undertaken in the summer, when data regarding the number of times (and in what capacity) participants have volunteered will be examined.

\subsection{Highlighting Public Engagement Impact}

To embed a culture of PE with SCD, the impact of successful engagement must be highlighted at all levels. To highlight PE within the department, the SCD PE Committee produces a monthly PE e-Newsletter to raise awareness of the Public Engagement work already being undertaken by staff and identifies opportunities for staff members who have not previously been a part of SCD's PE efforts to get involved. The committee also ensures PE is celebrated in the department, by producing content for department wide meetings. To highlight SCD's PE efforts within STFC, sections of these e-Newsletters are turned into articles for the STFC Intranet, with the help of SCD Impact team. These articles are subsequently turned into pieces for the external website [21-23] and are included in high level reports on the success of the department [17-19].

\subsection{Public Engagement as Continual Professional Development}

PE is often rightly cited as an opportunity for STFC staff to develop their communication skills, providing opportunities to adapt their explanations to audiences with different levels of science understanding, develop their skills to engage people with their science and get the public engaged with their science. In addition, PE allows STFC staff to develop and use their creativity to develop new PE resources, and to improve their confidence by collaborating with different staff members and groups. In particular, supervising work experience students allows early career staff members the opportunity to develop a project independently and manage both the project and a school student (or team of students) to deliver the project

\section{Future}

Within STFC, SCD is often highlighted as a department with a strong culture of public engagement. SCD should do more to share the expertise and best practices it has learned with other STFC departments. The creation of UK Research 
and Innovation (UKRI), which STFC is a part of along with the six other UK research councils, provides opportunities to influence their emerging Public Engagement best practices across all fields of knowledge within UKRI.

SCD will build on its current PE training programme. More Data Centre tour guides will be recruited across the department, by targeting new starters in general, not just graduate recruits. The trialled Computing PE induction will be evaluated based on the number of times attendees volunteered for PE activities compared with a baseline of early career new starters in 2018. If the workshop is an effective way of training new PE volunteers it will be repeated, potentially open to anyone in STFC with an interest in Computing PE.

SCD needs to link the impact of its PE efforts to projects within the department. Many of these projects have to show social or cultural impact of their work and PE is one way this can be done. However many of these projects are not currently capturing the PE effort that their staff undertake and as such do not have the necessary data to make the case that impact has been delivered.

As part of an evaluation of SCD's PE programme, the audience SCD engages with will be evaluated and the programme adjusted accordingly to better engage with its target audience. This will take effort, and might mean SCD deliver fewer activities directly, but will maximise the overall reach and impact.

\section{Summary}

UKRI/STFC's SCD has worked to embed a culture of PE with the department. SCD has its own director-endorsed PE strategy which compliments the STFC PE strategy and concentrates the department's effort to four main target audiences where SCD feel they can have the biggest impact. SCD works with the STFC PE teams to deliver its PE programme and they share frameworks where beneficial. One such framework is the Public Engagement Evaluation Framework [20], which the SCD PE Committee has adopted to evaluate its activities, which is the first step in an external review project of SCD's programme as a whole. The SCD PE committee ensures the PE efforts of the department are recognised and celebrated through departmental e-Newsletters, STFC wide news items and external reports [17-19]. To build capacity for engagement with the department SCD focuses on early career new starters as they generally have the time and management buy-in to deliver significant amounts of engagement. Staff new to SCD PE activities are being trained in an increasingly more systematic approach, such as the running of workshops dedicated to "train the trainer" style introductions to SCD's three core PE workshops allowing us to build on our successes and evolve the programme further in the future.

\section{References}

1. Science and Technology Facilities Council, "Types of STFC Research Funding," 04 March 2019. [Online]. Available: https://stfc.ukri.org/research-grants-handbook/4-types-of-stfc-research-funding/. [Accessed 04 February 2020].

2. Science and Technology Facilities Council, "Where We Work," 16 May 2017. [Online]. Available: https://stfc.ukri.org/about-us/where-we-work/. [Accessed 27 February 2020].

3. Science and Technology Facilities Council, "Corporate Strategy 2010 - 2020," 2010. [Online]. Available: https://stfc.ukri.org/files/corporate-publications/corporate-strategy-2010-20/. [Accessed 04 February 2020].

4. Science and Technology Facilities Council, "Inspiring Stories," 13 June 2018. [Online]. Available: https://stfc.ukri.org/public-engagement/inspiring-stories/. [Accessed 04 February 2020].

5. Science and Technology Facilities Council, "RAL Tier1," 15 December 2019. [Online]. Available: https://www.gridpp.ac.uk/wiki/RAL_Tier1. [Accessed 04 February 2020].

6. Science and Technology Facilities Council, "CEDA | JASMIN," [Online]. Available: https://www.ceda.ac.uk/services/jasmin/. [Accessed 04 February 2020].

7. Science and Technology Facilities Council, "Hartree Centre," [Online]. Available: https://www.hartree.stfc.ac.uk/Pages/home.aspx. [Accessed 27 February 2020].

8. Science and Technology Facilities Council, "SCD About us," [Online]. Available: https://www.scd.stfc.ac.uk/Pages/About-Us.aspx. [Accessed 27 February 2020].

9. CBI \& Pearson, “The Right Combination: CBI/Pearson Education and Skills Survey 2016," 19 July 2016. [Online]. Available: https://epale.ec.europa.eu/sites/default/files/cbi-education-and-skills-survey2016.pdf. [Accessed 27 February 2020].

10. Science and Technology Facilities Council, "Strategic Context \& Future Opportunities," 26 July 2018. [Online]. Available: https://stfc.ukri.org/files/corporate-publications/strategic-context/. [Accessed 27 February 2020].

11. CERN Education, Communication and Outreach group, "CERN's Communications Strategy 2017-2020," 2017. [Online]. Available: https://international-relations.web.cern.ch/sites/internationalrelations.web.cern.ch/files/files/strategy/CERN_CommunicationsStrategy_2017.pdf. [Accessed 27 February 2020].

12. Science and Technology Facilities Council, "Inspiring and Involving," 2016. [Online]. Available: https://stfc.ukri.org/files/corporate-publications/stfc-public-engagement-strategy/. [Accessed 27 February 2020]. 
13. Science and Technology Facilities Council, "Public Engagement Legacy Awards," 25 March 2019. [Online]. Available: https://stfc.ukri.org/public-engagement/public-engagement-grants/pe-funding-opportunities/pe-legacyawards/. [Accessed 27 January 2020].

14. National Co-ordinating Centre for Public Engagement, "Science Capital," 2017. [Online]. Available: https://www.publicengagement.ac.uk/nccpe-projects-and-services/nccpe-projects/national-forum-publicengagement-stem/science-capital. [Accessed 28 January 2020].

15. Science and Technology Facilities Council, "The Wonder initiative," 16 April 2019. [Online]. Available: https://stfc.ukri.org/public-engagement/wonder/. [Accessed 28 January 2020].

16. G. Corbett, G. Ryall, S. Palmer, I. P. Collier, J. Adams and R. Appleyard, "Public Outreach at RAL: Engaging the Next Generation of Scientists and Engineers," in Journal of Physics, 2015.

17. Scientific Computing Department, "Science Highlights 2015-16," 2016. [Online]. Available: https://www.scd.stfc.ac.uk/Pages/SCD-Science_Highlights_2016.pdf. [Accessed 27 February 2020].

18. Scientific Computing Department, "Science Highlights 2017," 2017. [Online]. Available: https://www.scd.stfc.ac.uk/Pages/SCD_Science_Highlights_2017.pdf. [Accessed 27 February 2020].

19. Scientific Computing Department, "Science Highlights 2018," 2018. [Online]. Available: https://www.scd.stfc.ac.uk/Pages/Science_Highlights_2017_2018.pdf. [Accessed 27 February 2020].

20. Science and Technology Facilities Council, "Public Engagement Evaluation Framework," April 2017. [Online]. Available: https://stfc.ukri.org/files/corporate-publications/public-engagement-evaluation-framework/. [Accessed 27 February 2020].

21. M. O'Sullivan, "Celebrating the Moon Landings of 1969 with (perhaps) the computer scientists of tomorrow," 23 July 2019. [Online]. Available: https://www.scd.stfc.ac.uk/Pages/Celebrating-the-Moon-Landings-of-1969-with(perhaps)-the-computer-scientists-of-tomorrow.aspx. [Accessed 31 January 2020].

22. M. O'Sullivan, “Summer Coding School 2019," 12 August 2019. [Online]. Available: https://www.scd.stfc.ac.uk/Pages/Summer-Coding-School.aspx. [Accessed 31 January 2020].

23. E. Greeves, “Ada Lovelace Day 2019,” 11 October 2019. [Online]. Available: https://www.scd.stfc.ac.uk/Pages/AdaLovelace-Day-2019.aspx. [Accessed 31 January 202].

24. Science and Technology Facilities Council, "ISIS Neutron and Muon Source," [Online]. Available: https://www.isis.stfc.ac.uk/Pages/home.aspx. [Accessed 04 February 2020].

25. Science and Technology Facilities Council, "Central Laser Facility," [Online]. Available: https://www.clf.stfc.ac.uk/Pages/home.aspx. [Accessed 04 February 2020].

26. Science and Technology Facilities Council, "RAL Space," [Online]. Available: https://www.ralspace.stfc.ac.uk/Pages/home.aspx. [Accessed 04 February 2020]. 